\title{
PLANT SPECIES RECOGNITION USING SPATIAL CORRELATION BETWEEN THE LEAF MARGIN AND THE LEAF SALIENT POINTS
}

\author{
Sofiene Mouine $\quad$ Itheri Yahiaoui ${ }^{1,2} \quad$ Anne Verroust-Blondet ${ }^{1}$ \\ ${ }^{1}$ INRIA Paris-Rocquencourt. Domaine de Voluceau 78153 Le Chesnay, France. \\ ${ }^{2}$ CReSTIC Université de Reims, France.
}

\begin{abstract}
In this paper, we propose an automatic approach for plant species identification, based on the visual information provided by the plant leaves. More precisely, we consider two sources of information: the leaf margin and the leaf salient points. We investigate two shape context based descriptors: the first one describes the leaf boundary while the second descriptor represents the spatial correlation between salient points of the leaf and its margin. We also study the performance of the fusion of these two descriptors on the ImageCLEF 2011 and 2012 leaf datasets. Experiments show the effectiveness and the efficiency of the proposed method.
\end{abstract}

Index Terms - Plant species identification, shape context, leaf shape, local descriptors, spatial relationships.

\section{INTRODUCTION}

Plant species identification is a topical issue in ecology, in particular in the botanical field. Plants are responsible for the presence of oxygen and play a key role in the food chain. Thus, there is a real need to identify plant species in order to preserve biological diversity. Botanists usually observe the leaves and other organs of a plant to determine its species. The leaf contains some of the most important features used in a plant identification task: leaf type (simple or compound), shape, color, texture, venation and margin. Furthermore, images of leaves can be easily acquired with either a flat-bed scanner or a digital camera. Many computer vision systems for plant species identification work on leaf databases $[1,3,5,6,15,18]$.

Apart from Nam et al. [21], where the shape features computed from the leaf margin are enriched with venation features, most approaches are based on the description of the leaf shape (see [7] for a review).

In [8], shape features are extracted using a set of morphological characters such as: Rectangularity, Sphericity, Circularity, etc. Eccentricity is used in the two-stage approach of Wang et al. [23] and of Caballero and Aranda [6] to reduce the search space.

Shape feature extraction techniques [17] have been adapted to the particular case of leaves, as for example, the Curvature Scale Space [6, 19] and Fourier-based descriptors [22, 26]. Shape context [4] and inner shape context $[3,16]$ techniques have proven their efficiency for leaf image retrieval. To describe the boundary of a shape accurately and obtain good retrieval results, a regular sampling of the contour points is computed. Then a large number of histograms are computed and compared, making the overall technique expensive. To solve this problem, Xie et al. [24] introduced the skeletal context, which uses a medial axis transform to produce an optimal sampling of the shape contour with a smaller number of points. In [20], the points that vote in the shape context (voting points) and the points where the shape context histograms are computed (computing points), are separated into two different sets. A low cardinality of the computing set reduces the computational cost while preserving and sometimes increasing the shape matching precision.

Shape and texture descriptors on oriented patches centered around Harris points are computed in [11]. No prior segmentation is made and Harris points are not necessarily located on the leaf margin. This generic approach obtained good results on scans of leaves in 2011 [12]. However, it is closely dependent on the image quality and the noise that may exist when acquiring the leaf images.

In this paper, we want to focus on our participation in the ImageCLEF 2012 plant identification task, where we obtained the best identification score on scan-like images. In addition, we extend our shape-context method [20] in order to retain more accurately two different sources of information: the leaf shape and the internal information (venation and texture). For this purpose, we study the performance of the fusion of two shape context based descriptors: $S C 0$ and $S C 2$ introduced in [20]. The $S C 0$ descriptor provides a good description of the leaf boundary and the $S C 2$ scenario computes the spatial relationships between the salient points and the leaf margin. Our approach is presented in details in Section 2. Evaluation results on scans and scan-like leaf images of the ImageCLEF 2011 and ImageCLEF 2012 plant identification tasks $[9,10]$ are reported and discussed in Section 3. 


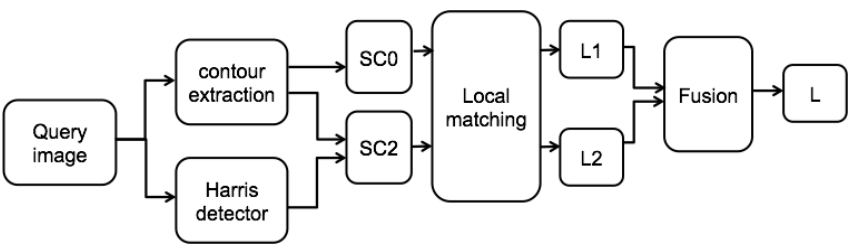

Fig. 1. The retrieval process. $L 1$ and $L 2$ are the lists of the most similar images to the query image computed respectively with $S C 0$ and $S C 2$ and $L$ is the fused list of results.

\section{OUR APPROACH}

As mentioned above, we believe that both contour and local interest points descriptions are useful for the leaf species identification. Our overall retrieval process, summarized in Figure 1, uses the advanced shape context features $S C 0$ and $S C 2$, based on the computation of spatial relationships between points of the leaf. These two features are briefly presented in section 2.1. The matching and the fusion steps are respectively described in sections 2.2 and 2.3 .

\subsection{Advanced shape context}

Given a set of $n$ points $\mathcal{V}$ and a point $p$ of $\mathbb{R}^{2}$, the advanced shape context of $\mathcal{V}$ on $p$ is a discrete representation of the set of $n$ vectors defined by the pairs of points $(p, q)$ with $q \in \mathcal{V}$. It is represented by a coarse histogram $a S C(p, \mathcal{V})$ where each pair of points $(p, q)$, represented by a radius $r$ and an angle $\theta$, contributes to the bin $k$ using the log-polar quantization introduced in [4].

$$
a S C(p, \mathcal{V})_{k}=\#\left\{q \in \mathcal{V}: q-p \in \operatorname{bin}_{p}(k)\right\}
$$

The set $\mathcal{V}$ is denoted the voting set of points and the set $\mathcal{C}$ of points $p$ of $\mathbb{R}^{2}$, where the advanced shape context $a S C(p, \mathcal{S})$ is computed, is called the computing set.

Two different scenarios, proposed in [20], are considered here:

- Scenario $S C 2$ represents the salient points, in the context defined by the leaf margin (cf. Figure 2(a)). The voting set of points $\mathcal{V}$ is composed of all the margin points. To approximate salient points of the leaf, Harris points are computed. They form the computing set $\mathcal{C}$.

- Scenario $S C 0$ captures the spatial relations between margin points. It corresponds to the shape context description of Belongie et al. [4]. The computing set $\mathcal{C}$ and the voting set $\mathcal{V}$ are equal to the leaf margin points, i.e. $n$ points extracted from the leaf boundary by a uniform quantization (cf. Figure 2(b)).

\subsection{Matching Method}

For $S C 0$ and $S C 2$, the feature matching process is the same. It is done by an approximate similarity search technique based

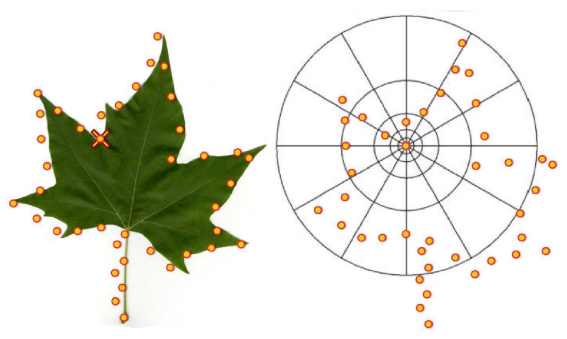

(a)

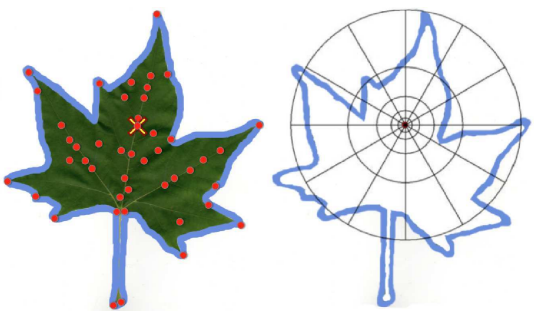

(b)

Fig. 2. Detected points on the leaves in $S C 0$ and $S C 2$ (a) Sample points on the leaf margin used in $S C 0$ where $\mathcal{C}=\mathcal{V}$ (b) Harris points in red (computing set) and contour points in blue (voting points), used in $S C 2$.

on a Locality Sensitive Hashing (LSH) method [13]. We use the distance $L_{2}$ to compute the similarity between two feature vectors. The principle of this algorithm is to project all the features in an $\mathrm{L}$ dimensional space and to use hash functions to reduce the search and the cost time. At query time, the features $F_{1}, F_{2}, \ldots, F_{n}$ of the query image are mapped onto the hash tables and the $k$-nearest neighbours of each feature $F_{i}$ are searched for in the buckets associated to $F_{i}$. These $n$ lists of candidate feature matches are used as input for a voting system to rank images according to the number of matched features.

\subsection{Fusion Method}

As illustrated in Figure 1, the descriptors $S C 0$ and $S C 2$ are computed independently. Their combination is done by a late fusion on the feature similarity ranking lists corresponding to the image query. The fusion of the two lists composed of the 30 first results is performed by the Leave Out algorithm (LO) described in [14].

\section{RESULTS}

Our descriptors have been tested on two leaf datasets: the scans and the scan-like images of the ImageCLEF datasets in 2011 [9] and in 2012 [10]. In all the experiments, a leaf image contains a single leaf on a uniform background. A preprocessing step is required to isolate the leaf area. First, we apply the Otsu threshold method to remove the background 
and keep only the mask corresponding to the leaf. A closed contour is then extracted from the leaf mask. Note that $S C 0$ takes as input a sequence of $n$ boundary points regardless of other leaf features such as texture, color and venation. The input for $S C 2$ is jointly the contour points (voting points) and the salient points (the computing set).

\subsection{Comparison with ImageCLEF 2011 results}

Let us now introduce the context of the plant identification task of ImageCLEF 2011[9].

The ImageCLEF 2011 dataset contains three categories of images:

- scans of leaves acquired using a flat-bed scanner,

- scan-like leaf images acquired using a digital camera,

- free natural photos.

For each category, the leaf images are divided into two sets: a training set and a testing set. The goal of the task is to find the correct tree species of each test image, knowing the species of the training images.

The identification score $S$ is quite different from the classic metrics. Two assumptions guided its definition:

- Leaves from the same tree may be more similar than leaves from different trees (the classification rate on each individual plant is averaged).

- Photos taken by the same person will have nearly the same acquisition protocol ( $S$ measures the mean of the average classification rate per user).

\begin{tabular}{|c|c|c|}
\hline run_id & Scans & Scan-like \\
\hline IFSC_USP_run2 & 0.562 & 0.402 \\
inria_imedia_plantnet_run1 & $\mathbf{0 . 6 8 5}$ & 0.464 \\
IFSC_USP_run1 & 0.411 & 0.430 \\
LIRIS_run3 & 0.546 & 0.513 \\
LIRIS_run1 & 0.539 & $\mathbf{0 . 5 4 3}$ \\
Sabanci-okan-run1 & $\mathbf{0 . 6 8 2}$ & 0.476 \\
LIRIS_run2 & 0.530 & 0.508 \\
LIRIS_run4 & 0.537 & $\mathbf{0 . 5 3 8}$ \\
inria_imedia_plantnet_run2 & 0.477 & $\mathbf{0 . 5 5 4}$ \\
IFSC_USP_run3 & 0.356 & 0.187 \\
DFH+GP [25] & $\mathbf{0 . 7 7 8}$ & $\mathbf{0 . 7 2 5}$ \\
\hline$S C 2$ & 0.676 & 0.677 \\
$S C 0$ & 0.654 & 0.706 \\
$S C 2+S C 0$ & $\mathbf{0 . 7 8 5}$ & 0.705 \\
\hline \multicolumn{2}{|c}{}
\end{tabular}

Table 1. Normalized classification scores of the scans and scan-like images on the ImageCLEF 2011 dataset using the evaluation metric of [9]

Then, $S$ is defined as follows:

$$
S=\frac{1}{U} \sum_{u=1}^{U} \frac{1}{P_{u}} \sum_{p=1}^{P_{u}} \frac{1}{N_{u, p}} \sum_{p=1}^{N_{u, p}} s_{u, p, n}
$$

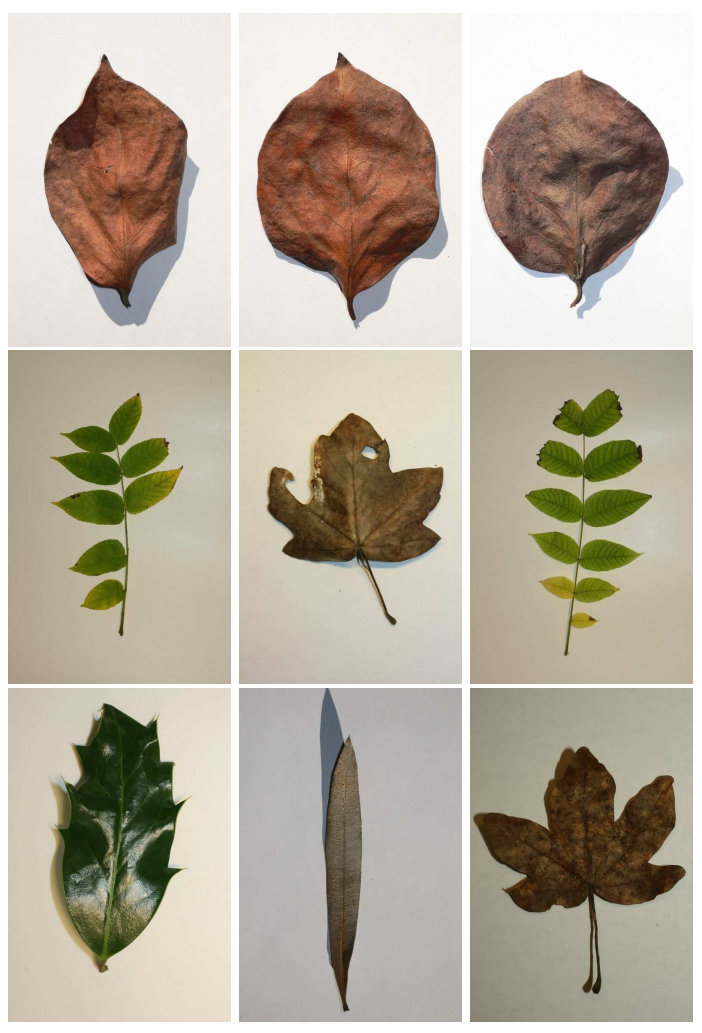

Fig. 3. Examples of scan-like images from the testing set of ImageCLEF 2011 dataset.

$U$ : number of users (who have at least one image in the test data).

$P_{u}$ : number of individual plants observed by the $u^{t h}$ user.

$N_{u, p}$ : number of pictures taken of the $p^{t h}$ plant observed by the $u^{t h}$ user.

$s_{u, p, n}$ : classification score ( 1 or 0 ) for the $n^{t h}$ picture taken of the $p^{\text {th }}$ plant observed by the $u^{\text {th }}$ user.

We focus on scans and scan-like images. The first category contains 2349 images for training and 721 test images. For the scan-like category, 717 images are used for training and 180 images for testing.

Table 1 shows the identification scores of our descriptors compared to other submitted runs of ImageCLEF 2011. SC2 performs better than $S C 0$ on scans. This is not the case on the scan-like images. This can be explained by the fact that the salient points are more relevant on scans because of the noise affecting scan-like images. The noise may be due to uneven lighting conditions, dead leaves and partial occlusion caused by leaf diseases. Let us examine the scan-like images of Figure 3. The first row shows examples of dead leaves. In fact, dead leaves become rigid and shrivelled, which may cause shadows in the acquisition protocol. Moreover, the visible damage on dead leaves affects the source of saliency like venation and texture. In the middle row, we show three scan-like images from the testing set, where parts of the leaf 
are missing. This is sometimes due to the falling leaflets of a compound leaf (first image in the middle row). Leaf diseases can be also the origin of torn leaves. Uneven lighting conditions are illustrated on the leaves of the third row.

The combination of $S C 0$ and $S C 2$, denoted by $S C 2+S C 0$, significantly improves the identification score on scans and outperforms all the other methods. It has the second best score on scan-like images. However, the combination here obtains almost the same score as $S C 0$.

The following parameters are used in this experiment:

- $S C 0$ : 50 contour points for both scans and scan-like images.

- $S C 2$ : 50 salient points for both scans and scan-like images.

- SC2 + SC0: 50 and 100 boundary points are used in $S C 0$ respectively for the scans and the scan-like images. 50 salient points are used in $S C 2$.

\subsection{Comparison with ImageCLEF 2012 results}

The formula used to rank the runs in the ImageCLEF 2012 plant identification task [10] is nearly the same as in 2011 (see [10] for details). The scan dataset contains 4870 images for training and 1760 test images. The scan-like category contains 1819 images for training and 907 images for testing. We participated in the ImageCLEF 2012 task where we obtained the best identification score on scan-like images [2]. with $S C 0$. We also proposed a method for the scans, which is a combination of $S C 2$ and three local features. In the current work, we keep only the score obtained by $S C 2$. The results presented in Table 2 confirm that $S C 2$ is not suitable for scan-like images. However, the information extracted by $S C 0$ and the one computed by $S C 2$ are complementary: the combination of $S C 0$ and $S C 2$ achieves the best score on the scan-like images and outperforms all the other methods. The performance on the scans is also improved by $S C 2+S C 0$. The identification score on scans is equal to the best score of the participating runs of ImageCLEF 2012.

We used the following parameters:

- SC0: 200 contour points for both scans and scan-like images.

- $S C 2$ : 50 salient points for scan-like images and 100 points for scans.

- $S C 2+S C 0$ : for $S C 2,50$ salient points for the scan-like images and 100 salient points for scans. SC0 used 200 contour points for both scans and scan-like images.

\section{CONCLUSION}

Two shape context based descriptors have been presented and combined for plant species identification.

- The first one gives a description of the leaf margin.

- The second one computes the spatial relations between the salient points and the leaf contour points.

The experiments carried out on ImageCLEF leaf datasets,

\begin{tabular}{|c|c|c|}
\hline & Scans & Scan-like \\
\hline Top 3 Scores & 0.58 & 0.59 \\
& 0.49 & 0.55 \\
& 0.47 & 0.54 \\
\hline$S C 0$ & 0.52 & $\mathbf{0 . 5 9}$ \\
$S C 2$ & 0.51 & 0.42 \\
$S C 2+S C 0$ & $\mathbf{0 . 5 8}$ & $\mathbf{0 . 6 1}$ \\
\hline
\end{tabular}

Table 2. Normalized classification scores of the scans and scan-like images using the evaluation metric of [10] (ImageCLEF 2012)

show the complementarity of the two descriptions $S C 0$ and $S C 2$. Their combination generally improves the identification score.

Starting from the assumption that the location of salient points could be approximated with a corner edge detector, we use the Harris detector. This step can be improved by developing a specific detector of key points of the venation network. Work in progress also focuses on the fusion of $S C 2$ with other types of shape descriptors.

\section{Acknowledgements}

This research has been conducted with the support of the Agropolis Foundation through the Pl@ntNet project. We would like also to thank Vera Bakić for her help to accomplish this work and Richard James for revising the English of this paper.

\section{REFERENCES}

[1] A. R. Backes, D. Casanova, and O. M. Bruno. A complex network-based approach for boundary shape analysis. Pattern Recognition, 42(1):54-67, 2009.

[2] V. Bakic, I. Yahiaoui, S. Mouine, S. Litayem Ouertani, W. Ouertani, A. Verroust-Blondet, H. Goëau, and A. Joly. Inria IMEDIA2's Participation at ImageCLEF 2012 Plant Identification Task. In CLEF (Online Working Notes/Labs/Workshop), Rome, Italy, Sept. 2012.

[3] P. Belhumeur, D. Chen, S. Feiner, D. Jacobs, W. Kress, H. Ling, I. Lopez, R. Ramamoorthi, S. Sheorey, S. White, and L. Zhang. Searching the world's herbaria: A system for visual identification of plant species. In European Conference on Computer Vision (ECCV), pages 116-129, 2008.

[4] S. Belongie, J. Malik, and J. Puzicha. Shape matching and object recognition using shape contexts. IEEE Transactions on Pattern Analysis and Machine Intelligence, 24(4):509 -522, apr 2002. 
[5] O. M. Bruno, R. de Oliveira Plotze, M. Falvo, and M. de Castro. Fractal dimension applied to plant identification. Information Sciences, 178(12):2722 - 2733, 2008.

[6] C. Caballero and M. C. Aranda. Plant species identification using leaf image retrieval. In ACM International Conference on Image and Video Retrieval (CIVR), pages 327-334, 2010.

[7] J. S. Cope, D. Corney, J. Y. Clark, P. Remagnino, and P. Wilkin. Plant species identification using digital morphometrics: A review. Expert Systems with Applications, 39(8):7562 - 7573, 2012.

[8] J.-X. Du, X.-F. Wang, and G.-J. Zhang. Leaf shape based plant species recognition. Applied Mathematics and Computation, 185(2):883 - 893, 2007.

[9] H. Goëau, P. Bonnet, A. Joly, N. Boujemaa, D. Barthelemy, J.-F. Molino, P. Birnbaum, E. Mouysset, and M. Picard. The CLEF 2011 plant images classification task. In CLEF (Notebook Papers/Labs/Workshop), 2011.

[10] H. Goëau, P. Bonnet, A. Joly, I. Yahiaoui, D. Barthélémy, N. Boujemaa, and J.-F. Molino. The IMAGECLEF 2012 Plant identification Task. In CLEF 2012, Rome, Italy, Sept. 2012.

[11] H. Goëau, A. Joly, S. Selmi, P. Bonnet, E. Mouysset, L. Joyeux, J.-F. Molino, P. Birnbaum, D. Barthélémy, and N. Boujemaa. Visual-based plant species identification from crowdsourced data. In 19th ACM international conference on Multimedia, pages 813-814, 2011.

[12] H. Goëau, A. Joly, I. Yahiaoui, P. Bonnet, and E. Mouysset. Participation of INRIA\& Pl@ntNet to ImageCLEF 2011 plant images classification task. In CLEF (Notebook Papers/Labs/Workshop), 2011.

[13] A. Joly and O. Buisson. A posteriori multi-probe locality hashing. In 16th ACM international conference on Multimedia, pages 209-218, 2008.

[14] M. Jović, Y. Hatakeyama, F. Dong, and K. Hirota. Image retrieval based on similarity score fusion from feature similarity ranking lists. In Third international conference on Fuzzy Systems and Knowledge Discovery (FSKD’06), pages 461-470, 2006.

[15] N. Kumar, P. N. Belhumeur, A. Biswas, D. W. Jacobs, W. J. Kress, I. C. Lopez, and J. V. B. Soares. Leafsnap: A computer vision system for automatic plant species identification. In 12th European Conference on Computer Vision (ECCV 2012), pages 502-516, Florence, Italy, Oct. 2012.
[16] H. Ling and D. Jacobs. Shape classification using the inner-distance. IEEE Transactions on Pattern Analysis and Machine Intelligence, 29(2):286 -299, Feb. 2007.

[17] Y. Mingqiang, K. Kidiyo, and R. Joseph. A survey of shape feature extraction techniques. Pattern Recognition Techniques, Technology and Applications, 2008. ISBN: 978-953-7619-24-4, InTech.

[18] F. Mokhtarian and S. Abbasi. Matching shapes with self-intersections: application to leaf classification. IEEE Transactions on Image Processing, 13(5):653 661, May 2004.

[19] F. Mokhtarian, S. Abbasi, and J. Kittler. Robust and efficient shape indexing through curvature scale space. In British Machine Vision Conference (BMVC), 1996.

[20] S. Mouine, I. Yahiaoui, and A. Verroust-Blondet. Advanced shape context for plant species identification using leaf image retrieval. In 2nd ACM International Conference on Multimedia Retrieval (ICMR 2012), pages 49:1-49:8, 2012.

[21] Y. Nam, E. Hwang, and D. Kim. A similarity-based leaf image retrieval scheme: Joining shape and venation features. Computer Vision and Image Understanding, 110(2):245 - 259, 2008.

[22] J. C. Neto, G. E. Meyer, D. D. Jones, and A. K. Samal. Plant species identification using elliptic Fourier leaf shape analysis. Computers and Electronics in Agriculture, 50(2):121 - 134, 2006.

[23] Z. Wang, Z. Chi, and D. Feng. Shape based leaf image retrieval. IEE Proceedings on Vision, Image and Signal Processing, 150(1):34 - 43, Feb. 2003.

[24] J. Xie, P.-A. Heng, and M. Shah. Shape matching and modeling using skeletal context. Pattern Recogn., 41(5):1756-1767, May 2008.

[25] I. Yahiaoui, O. Mzoughi, and N. Boujemaa. Leaf shape descriptor for tree species identification. In Proceedings of the 2012 IEEE International Conference on Multimedia and Expo, ICME 2012, pages 254-259, 2012.

[26] C. T. Zahn and R. Z. Roskies. Fourier descriptors for plane closed curves. IEEE Trans. Comput., 21(3):269281, Mar. 1972. 